\title{
Altered expression of microRNA-92b-3p predicts survival outcomes of patients with prostate cancer and functions as an oncogene in tumor progression
}

\author{
GANG WANG ${ }^{*}$, BO CHENG* ${ }^{*}$ RENFENG JIA, BO TAN and WENLONG LIU \\ Department of Urology, Shengli Oilfield Central Hospital, Dongying, Shandong 257034, P.R. China
}

Received April 9, 2020; Accepted October 12, 2020

DOI: $10.3892 / \mathrm{ol} .2020 .12265$

\begin{abstract}
The global incidence of prostate cancer (PCa) has been increasing in recent years. Meanwhile, some studies have indicated the association between malignancies, such as lung and gastric cancer and $\mathrm{PCa}$, and microRNAs (miRNAs). The present study was designed to assess the prognostic value of miR-92b-3p in patients with PCa and further investigate the biological function of miR-92b-3p. Real-time quantitative polymerase chain reaction was used to estimate the expression of miR-92b-3p in PCa tissues and cell lines compared with normal tissues and cells. Kaplan-Meier method was used to analyze the overall survival rate of patients with PCa. A Cox regression analysis was used to verify the prognostic value of miR-92b-3p. The biological function of miR-92b-3p was investigated using cell experiments. The findings of the present study revealed the upregulated expression of miR-92b-3p in PCa tissues and cells compared with normal tissues and cells. The overexpression of miR-92b-3p was significantly associated with the distant metastasis status and Tumor-Node-Metastasis stage of patients with $\mathrm{PCa}$ and predicted poor prognosis of PCa. In addition, the cell experiment results indicated that miR-92b-3p overexpression in PCa cells promoted cell proliferation, migration and invasion. The present study revealed that the overexpression of miR-92b-3p predicted poor prognosis in patients with $\mathrm{PCa}$. Decreased expression of miR-92b-3p can suppress PCa cell proliferation, migration and invasion, which indicated that miR-92b-3p may function as an oncogene and serve as a novel therapeutic target for PCa.
\end{abstract}

Correspondence to: Dr Wenlong Liu, Department of Urology, Shengli Oilfield Central Hospital, 31 Jinan Road, Dongying, Shandong 257034, P.R. China

E-mail:1mhealth0402@163.com

${ }^{*}$ Contributed equally

Key words: microRNA-92b-3p, prostate cancer, prognosis, proliferation, migration, invasion

\section{Introduction}

Prostate cancer ( $\mathrm{PCa})$ is the second most frequent malignancy found in men worldwide (1). There were 1.1 million new cases of PCa in 2012, accounting for $15 \%$ of male cancers (2). The incidence of $\mathrm{PCa}$ has been increasing worldwide in recent years, particularly in Asian countries, such as China, Japan, Korea and Indian (3). Statistical data indicates that PCa is the sixth leading cause of cancer-related deaths that occur in men, with an estimated 307,000 deaths in 2015, which accounted for $6.6 \%$ of total male cancer-associated mortality $(4,5)$. There are some risk factors which may lead to PCa, such as obesity, smoking, alcohol consumption, a vasectomy and diet (6). Currently, early diagnosis and efficient treatment remain obstacles in PCa, owing to the unspecific clinical symptoms and complex disease pathogenesis (7). Although some advances have been made in tumor therapeutic strategies, such as surgery, chemotherapy and radiotherapy, the prognosis in patients with $\mathrm{PCa}$ remains poor (8). In addition, majority of patients with PCa suffer from severe pain, fractures and abnormal urination, which seriously reduce the quality of life of the patients (9). Therefore, it is necessary to develop novel reliable therapeutic strategies to improve the treatment of $\mathrm{PCa}$.

MicroRNAs (miRNAs) are a group of non-coding small RNAs, approximately 18-22 nucleotides in length, that are involved in numerous important cell processes, such as cell proliferation, migration, invasion, differentiation and apoptosis (10). miRNAs can directly bind the 3'-untranslated region of target genes leading to inhibition in gene expression (11). Emerging studies report that miRNAs serve important regulatory roles in tumor progression making them potential therapeutic targets in various human cancers, such as lung and liver cancer $(12,13)$. Notably, some aberrantly expressed miRNAs have also been detected in PCa, such as miR-215-5p (14) and miR-145 (15), which participate in disease pathogenesis and are associated with the prognosis of $\mathrm{PCa}$. miR-92b-3p has been investigated in some human malignancies in previous studies. For instance, Long et al (16) indicated that miR-92b-3p acted as a tumor suppressor in pancreatic cancer by targeting Gabra3-associated oncogenic pathways. Notably, a study by Ma et al (17) found an overexpression of miR-92b-3p in PCa cell lines, which was associated with PCa metastasis, but this study did not investigate the clinical significance and biological function of miR-92b-3p in PCa. 
To further improve PCa therapy, the present study aimed to detect the expression of miR-92b-3p in PCa tissues and cell lines and evaluate the clinical significance of miR-92b-3p. In addition, the biological function of miR-92b-3p was also investigated using gain- and loss-of-function experiments in PCa cells. The findings of the present study may provide a novel biomarker to predict $\mathrm{PCa}$ prognosis and a potential therapeutic target for improving the treatment of $\mathrm{PCa}$.

\section{Materials and methods}

Patients and tissue collection. A total of 108 patients (average age of $66.7 \pm 9.1$ years) who had been pathologically diagnosed as PCa in Shengli Oilfield Central Hospital (Dongying, China) from June 2010 to May 2013 were enrolled in the present study. PCa tissues and adjacent normal tissues $(>2-\mathrm{cm}$ from the edge of tumor) were obtained during resection and immediately stored in liquid nitrogen at $-80^{\circ} \mathrm{C}$ for further use. The patients were enrolled in accordance with the following inclusion criteria: i) The tumor tissues were histopathologically diagnosed with $\mathrm{PCa}$; ii) none of the patients had received any antitumor therapy prior to surgery; iii) the age range of the patients was 18-75 years; iv) had complete demographic and clinical data; and v) signed informed consent for the use of clinical samples and data. In addition, the following exclusion criteria were used: i) Cases with serious heart, liver, respiratory and kidney diseases; ii) cases with an age $<18$ or $>75$; and iii) cases that had incomplete clinical data or had no follow-up information. The collected PCa tissues were graded according to the Gleason grading system (18). In addition, the Tumor-Node-Metastasis (TNM) stage of the PCa tissues was determined using the criteria of the American Joint Committee on Cancer classification (19). In order to record the survival status of the patients, a 5-year follow-up survey was conducted, and each patient was followed up once a month by telephone. Among the 108 patients with PCa, 59 cases received antiandrogen therapy (flutamide) after surgery and 54 patients developed androgen-independent $\mathrm{PCa}$. All patients had signed an informed consent form and the protocol of this study received approval from the Ethics Committee of Shengli Oilfield Central Hospital (Dongying, China; approval no. SLYTh100219).

Cell culture and transfection. Four PCa cell lines DU145, LNCaP, VCaP, 22Rv1 and one human prostate epithelial cell line RWPE1 were purchased from the Type Culture Collection of the Chinese Academy of Sciences. The PCa cells were cultured in RPMI-1640 medium (BioTek China) supplemented with $10 \%$ fetal bovine serum (FBS; Thermo Fisher Scientific Inc.). RWPE1 cells were cultured in K-SMF medium (Gibco; Thermo Fisher Scientific Inc.) containing $5 \mathrm{ng} / 1$ epidermal growth factor (Gibco; Thermo Fisher Scientific Inc.) and $50 \mu \mathrm{g} / \mathrm{ml}$ bovine pituitary extract (Invitrogen; Thermo Fisher Scientific Inc.). All cells were maintained at $37^{\circ} \mathrm{C}$ in a humidified incubator with $5 \% \mathrm{CO}_{2}$.

Cell lines LNCaP and DU145 were selected to perform the transfection experiments due to significantly higher expression of miR-92b-3p in the two cell lines compared with the normal cells. To regulate the expression of miR-92b-3p, $50 \mathrm{nM}$ of miR-92b-3p mimic and mimic negative control (NC), and
$100 \mathrm{nM}$ of miR-92b-3p inhibitor and inhibitor NC were synthesized by Guangzhou RiboBio Co., Ltd.. The above sequences were separately transfected into PCa cells using Lipofectamine $2000^{\circledR}$ (Invitrogen; Thermo Fisher Scientific Inc.) at $37^{\circ} \mathrm{C}$ following the manufacturer's instructions. Untransfected cells were used as controls. The sequences of transfection vectors were as follows: miR-92b-3p Mimic 5'-UAUUGCACUUGU CCCGGCCUGU-3'; mimic NC 5'-UUCUCCGAACGUGUC ACGU-3'; miR-92b-3p inhibitor 5'-ACAGGCCGGGACAAG UGCAAUA-3'; inhibitor NC 5'-CAGUACUUUUGUGUA GUACAA-3'. After $48 \mathrm{~h}$ of cell transfection, the subsequent cell experiments were performed.

$R N A$ extraction and real-time quantitative $(R T-q) P C R$. Total RNA from the PCa tissues and all cell lines were extracted by using TRIzol ${ }^{\circledR}$ reagent (Invitrogen; Thermo Fisher Scientific Inc.) according to the manufacturer's instructions. cDNA synthesis was performed using the PrimeScript RT reagent kit (Takara Bio Inc.) according to the manufacturer's instructions. The expression of miR-92b-3p was assessed using RT-qPCR, which was performed with the SYBR Green I Master Mix kit (Invitrogen; Thermo Fisher Scientific Inc.) on a 7500 Real-Time PCR System (Applied Biosystems; Thermo Fisher Scientific Inc.) with following thermocycling conditions: $95^{\circ} \mathrm{C}$ For $10 \mathrm{~min}$ and 40 cycles of $95^{\circ} \mathrm{C}$ for $20 \mathrm{sec}$, $58^{\circ} \mathrm{C}$ for $15 \mathrm{sec}, 72^{\circ} \mathrm{C}$ for $20 \mathrm{sec}$. U6 was used as an internal control and the $2^{-\Delta \Delta \mathrm{Cq}}$ method (20) was used to calculate the final expression level of miR-92b-3p. The sequences of primers used were as follows: miR-92b-3p forward 5'-GCCGAGTAT TGCACTTGTCC-3', miR-92b-3p reverse 5'-CTCAACTGG TGTCGTGGA-3'; U6 forward 5'-CTCGCTTCGGCAGCA CA-3', U6 reverse 5'-AACGCTTCACGAATTTGCGT-3'.

Cell proliferation assay. DU145 and LNCaP cells were selected to perform the cell experiments following transfection with miR-92b-3p mimic, miR-92b-3p inhibitor or NCs as aforementioned. After the cells grew into a stable phase, they were seeded in 96-well plates at a density of $5 \times 10^{3}$ cells/well and cell proliferation was assessed using the MTT assay. The cells were incubated at $37^{\circ} \mathrm{C}$ for 3 days and $10 \mu \mathrm{MTT}(5 \mathrm{mg} / \mathrm{ml}$; Sigma-Aldrich; Merck KGaA) was added every $24 \mathrm{~h}$ followed by subsequent $4 \mathrm{~h}$ incubation at $37^{\circ} \mathrm{C}$. Subsequently, $150 \mu \mathrm{l}$ of DMSO was added to each well and the absorbance of cells was measured using a microplate reader at $570 \mathrm{~nm}$.

Cell migration and invasion assays. PCa cell migration and invasion abilities were measured using Transwell chambers (Corning Inc.). Membranes were precoated with Matrigel at $37^{\circ} \mathrm{C}$ for $6 \mathrm{~h}$ for invasion assay. Serum free RPMI-1640 medium without any drug treatment was added to the upper chambers and the lower chambers were filled with RPMI-1640 medium supplemented with 10\% FBS. DU145 and LNCaP cells $\left(5 \times 10^{5}\right.$ cells/well) were seeded in the upper chambers. Following $48 \mathrm{~h}$ incubation at $37^{\circ} \mathrm{C}$, the cells in the lower chambers were stained using $0.2 \%$ crystal violet for $10 \mathrm{~min}$ at room temperature and counted under an inverted light microscope (Olympus Corporation) with a magnification of x200.

Statistical analysis. Data in the present study was analyzed using SPSS 21.0 software (IBM Corp.) and GraphPad Prism 7.0 
Table I. Association of miR-92b-3p expression and clinicopathological features of 108 patients with PCa.

\begin{tabular}{|c|c|c|c|c|c|}
\hline \multirow[b]{2}{*}{ Features } & \multirow[b]{2}{*}{ Category } & \multirow[b]{2}{*}{ Total patients } & \multicolumn{2}{|c|}{ miR-92b-3p expression } & \multirow[b]{2}{*}{ P-value } \\
\hline & & & Low, $n=50$ & High, $n=58$ & \\
\hline \multirow[t]{3}{*}{ Age, years } & & & & & 0.685 \\
\hline & $<60$ & 41 & 20 & 21 & \\
\hline & $\geq 60$ & 67 & 30 & 37 & \\
\hline \multirow[t]{3}{*}{ PSA, ng/ml } & & & & & 0.009 \\
\hline & $<10$ & 34 & 22 & 12 & \\
\hline & $\geq 10$ & 74 & 28 & 46 & \\
\hline \multirow[t]{3}{*}{ Bone metastasis } & & & & & 0.033 \\
\hline & Negative & 55 & 31 & 24 & \\
\hline & Positive & 53 & 19 & 34 & \\
\hline \multirow[t]{3}{*}{ Gleason score } & & & & & 0.001 \\
\hline & $\leq 7$ & 59 & 36 & 23 & \\
\hline & $>7$ & 49 & 14 & 35 & \\
\hline \multirow[t]{3}{*}{ TNM stage } & & & & & 0.015 \\
\hline & I-II & 47 & 28 & 19 & \\
\hline & III-IV & 61 & 22 & 39 & \\
\hline
\end{tabular}

PSA, prostate-specific antigen; TNM, tumor-node-metastasis; PCa, prostate cancer.

software (GraphPad Software, Inc.) and were expressed as mean \pm SD. Each experiment was repeated at least three times. A Chi-square test was performed to analyze the association between miR-92b-3p and the clinicopathological characteristics of patients with PCa. The differences between groups were assessed using a paired Student's t-test or one-way ANOVA followed by a post hoc Tukey's test. The Kaplan-Meier method was used to generate the survival curves of patients with PCa and the differences between survival curves were analyzed using the log-rank test. The prognostic significance of miR-92b-3p was evaluated using Cox regression analysis. $\mathrm{P}<0.05$ was considered to indicate a statistically significant difference.

\section{Results}

miR-92b-3p is upregulated in PCa tissues and cell lines. As shown in Fig. 1A, the expression of miR-92b-3p in PCa tissues was significantly upregulated compared to adjacent normal tissues $(\mathrm{P}<0.001)$. Upregulated expression of miR-92b-3p was also found in $\mathrm{PCa}$ cell lines compared with normal human prostate epithelial cells $(\mathrm{P}<0.01$ or $\mathrm{P}<0.001$; Fig. 1B).

Association of miR-92b-3p with clinicopathological characteristics of patients with PCa. Since miR-92b-3p was found to be upregulated in PCa tissues and cells, it was hypothesized that miR-92b-3p may be related to PCa development. Therefore, the association between miR-92b-3p expression and clinicopathological characteristics of patients with $\mathrm{PCa}$ were investigated. For this analysis, patients with $\mathrm{PCa}$ were divided into a low miR-92b-3p expression group $(n=50)$ and high miR-92b-3p expression group $(n=58)$ based on the mean expression value (1.824). The data were analyzed using a
Table II. Multivariate analysis for overall survival of patients with PCa using the Cox regression model.

\begin{tabular}{lccc}
\hline Variables & HR & $95 \% \mathrm{CI}$ & P-value \\
\hline Age & 1.431 & $0.589-3.425$ & 0.421 \\
PSA & 1.715 & $0.845-3.503$ & 0.140 \\
Bone metastasis & 1.985 & $1.018-3.742$ & 0.049 \\
Gleason score & 2.161 & $1.069-4.370$ & 0.032 \\
TNM stage & 2.085 & $1.042-4.185$ & 0.040 \\
miR-92b-3p & 2.346 & $1.185-5.276$ & 0.025 \\
\hline
\end{tabular}

PSA, prostate-specific antigen; TNM, tumor-node-metastasis; $\mathrm{PCa}$, prostate cancer; HR, hazard ratio; CI, confidence interval.

Chi-square test and presented in Table I. The expression of miR-92b-3p was associated with prostate-specific antigen (PSA), bone metastasis, Gleason score and TNM stage (all $\mathrm{P}<0.05$; Table I). However, no relationship was found between miR-92b-3p and age ( $\mathrm{P}>0.05$; Table I).

Relationship between miR-92b-3p and overall survival of patients with PCa. Kaplan-Meier curves revealed that patients with higher miR-92b-3p expression had a shorter overall survival rate compared with those with lower miR-92b-3p expression ( $\log$-rank $\mathrm{P}=0.022$; Fig. $2 \mathrm{~A}$ ). In addition, the predictive value of miR-92b-3p for the prognosis of patients with $\mathrm{PCa}$ with different status of bone metastasis was evaluated. As shown in Fig. 2B and C, patients with PCa with high expression of miR-92b-3p had a shorter survival rate under both negative and positive metastasis status compared 
A

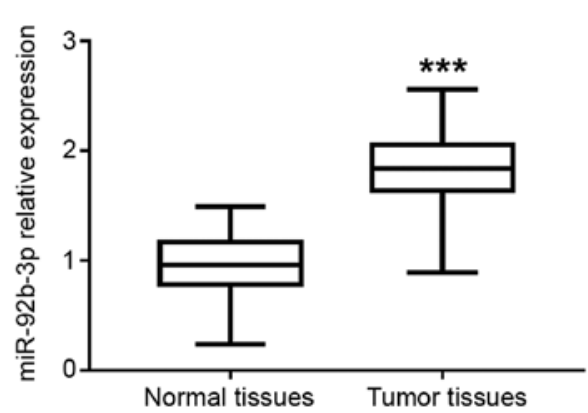

B

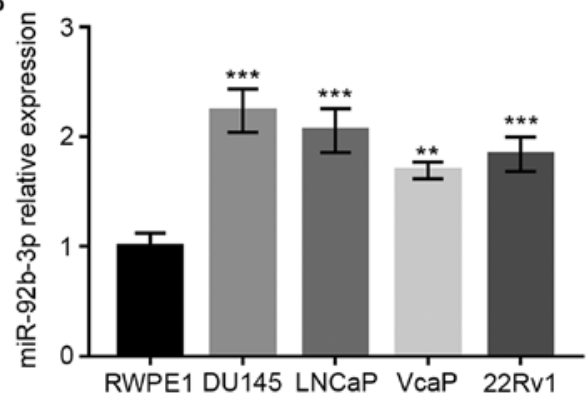

Figure 1. Expression of miR-92b-3p in patients with PCA and PCA cell lines. (A) Expression of miR-92b-3p in PCa tumor tissues was higher compared with that in adjacent normal tissues (the differences between the two groups were assessed using Student's t-test). (B) PCa cell lines had elevated miR-92b-3p expression compared with normal cells. ${ }^{* *} \mathrm{P}<0.01 ;{ }^{* * *} \mathrm{P}<0.001$. $\mathrm{PCa}$, prostrate cancer; miRNA, microRNA.
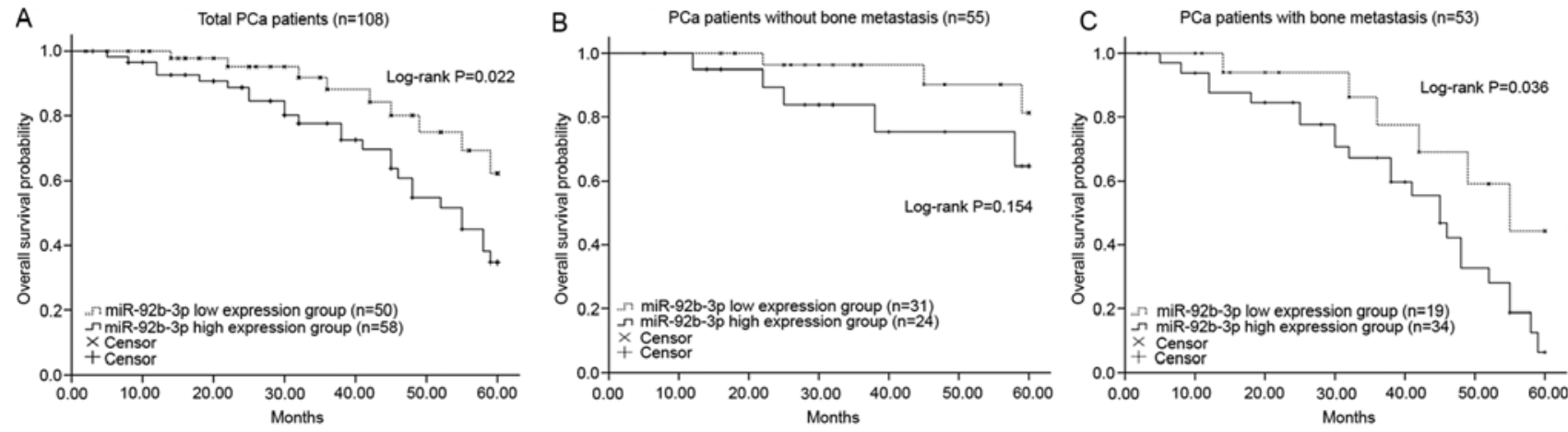

Figure 2. Survival analysis of patients with PCa using the Kaplan-Meier method. (A) High miR-92b-3p expression was associated with shorter overall survival time in PCa patients (log-rank $\mathrm{P}=0.022$ ). (B) Patients without bone metastasis had poor survival when the expression of miR-92b-3p was high, but the difference had no statistical significance (log-rank $\mathrm{P}=0.154)$. (C) In patients with bone metastasis, high miR-92b-3p was associated with poor overall survival $(\mathrm{P}=0.036)$. PCa, prostate cancer; miRNA, microRNA.

with those patients with low levels of miR-92b-3p, and high miR-92b-3p expression in patients with positive bone metastasis was significantly associated with poor overall survival (log-rank $\mathrm{P}=0.036$; Fig. 2C). However, the difference of survival time between high and low miR-92b-3p groups was not statistically significant in patients with negative bone metastasis (log-rank $\mathrm{P}=0.154$; Fig. 2B). Furthermore, the Cox regression analysis data revealed that the overexpression of miR-92b-3p was an independent prognostic factor for the overall survival rate of patients with $\mathrm{PCa}$ [hazard ratio $(\mathrm{HR})=2.346 ; 95 \%$ confidence interval $(\mathrm{CI})=1.185-5.276$; $\mathrm{P}$-value $=0.025$; Table II].

Overexpression of miR-92b-3p promotes $P C a$ cell proliferation. To further investigate the biological function of miR-92b-3p in PCa cells, cell experiments were conducted. Cell lines DU-145 and LNCaP were included in cell transfection as they had significantly high miR-92b-3p expression compared with the normal cell line RWPE1. The expression of miR-92b-3p was dramatically higher in cells transfected with miR-92b-3p mimic, while it was dramatically lower in cells transfected with miR-92b-3p inhibitor compared with the cells in control group (all $\mathrm{P}<0.001$; Fig. 3A and B). Using the MTT assay, it was found that the overexpression of miR-92b-3p promoted tumor cell proliferation, whereas downregulation of miR-92b-3p inhibited the cell proliferation of DU-145 and LNCaP cells (all $\mathrm{P}<0.05$; Fig. 3C and D).
Overexpression of $m i R-92 b-3 p$ promotes cell migration and invasion of PCa cells. Subsequently, Transwell chambers were used to measure the migration and invasion abilities of DU-145 and LNCaP cells. The migration ability of PCa cells was promoted by miR-92b-3p expression overexpression, while was inhibited by the downregulation of miR-92b-3p $(\mathrm{P}<0.05$ or $\mathrm{P}<0.01$; Fig. 4A). As for the invasion ability, we found that the overexpression of miR-92b-3p significantly boosted $\mathrm{PCa}$ cell invasion, while the downregulation of miR-92b-3p significantly reduced $\mathrm{PCa}$ cell invasion (all $\mathrm{P}<0.05$; Fig. 4B).

\section{Discussion}

There is growing evidence that indicates that miRNAs, which can transmit signals and regulate intracellular gene expression, serve important roles in tumor development and progression (21). Additionally, some studies have also found that miRNAs serve as tumor suppressors or oncogenes involved in tumor progression $(22,23)$. For example, a study by Wang et al (24) revealed that miR-66a-3p expression in gastric cancer tissues and cells was upregulated, which may function as an oncogene by targeting the Hippo pathway. Additionally, miR-506 was downregulated in cervical cancer tissues, which further showed that miR-506 was able to suppress tumor cell proliferation and can serve as a novel therapeutic target of cervical cancer (25). Similarly, in PCa tissues, some miRNAs with ectopic expression have also been found. For instance, 

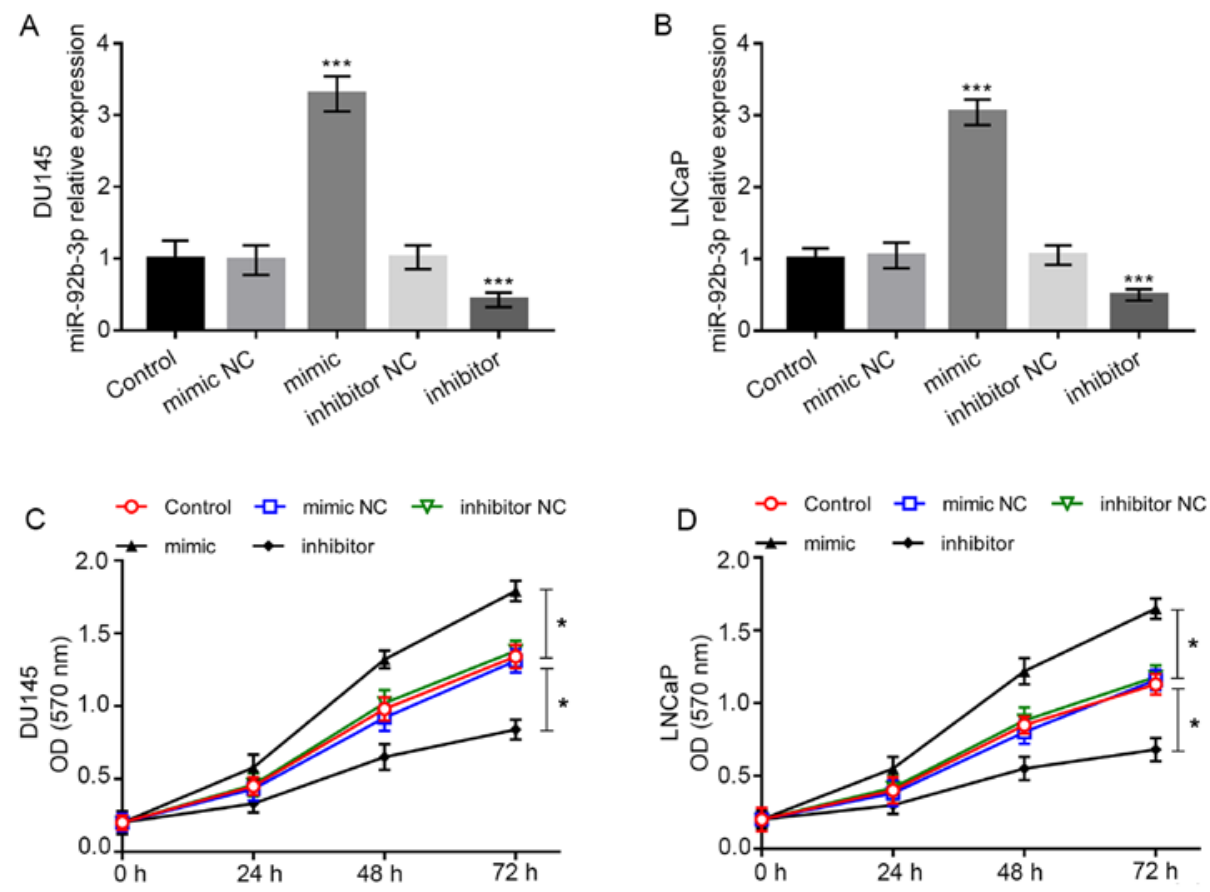

Figure 3. Regulatory effect of miR-92b-3p on cell proliferation of DU145 and LNCaP cells. (A and B) Expression of miR-92b-3p was upregulated by the miR-92b-3p mimic, but was downregulated by the miR-92b-3p inhibitor compared with cells in the control group. (C and D) Overexpression of miR-92b-3p in PCa cells led to increased cell proliferation, while the downregulation of miR-92b-3p inhibited cell proliferation. ${ }^{*} \mathrm{P}<0.05,{ }^{* * *} \mathrm{P}<0.001$ compared with the untransfected control group. PCa, prostate cancer; miRNA, microRNA; OD, optical density; NC, negative control.
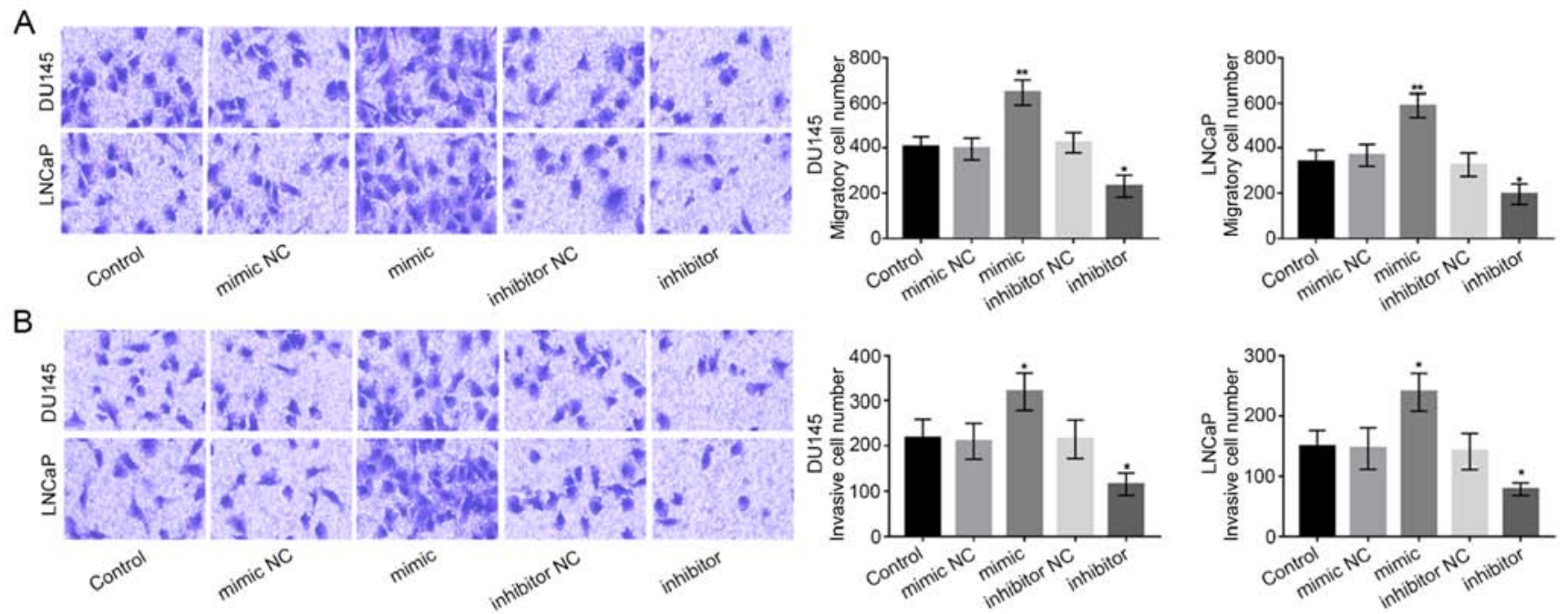

Figure 4. miR-92b-3p accelerated PCa cell migration and invasion abilities in DU145 and LNCaP cells. (A) PCa cell migration was enhanced by the overexpression of miR-92b-3p, but was inhibited by the silencing of miR-92b-3p. (B) The cell invasion was promoted in PCa cells with overexpression of miR-92b-3p, but was suppressed in cells with decreased miR-92b-3p. ${ }^{*} \mathrm{P}<0.05$ and ${ }^{* *} \mathrm{P}<0.01$ compared with untransfected control group. PCa, prostate cancer; miRNA, microRNA; NC, negative control.

a study by Zhang et al (26) found that downregulation of miR-410-3p can accelerate PCa cell apoptosis and suppress cell proliferation, migration and epithelial-mesenchymal transition progress and exert oncogenic functions by downregulating PTEN. A study also showed that the downregulation of miR-375 presented better discriminating performance compared with prostate-specific antigen indicating that miR-375 had stronger diagnostic accuracy and can be used as a non-invasive biomarker for PCa screening (27). The aforementioned studies demonstrated the importance of identifying novel miRNAs that affect tumor progression to improve the treatment of $\mathrm{PCa}$

The present study focused on the expression and functional role of miR-92b-3p in PCa. miR-92b-3p has been previously investigated in some cancers. For example, the overexpression of miR-92b-3p was detected in gastric cancer SGC-7901 cells, which inhibited SGC-7901 cell proliferation, migration and invasion via downregulating matrix metalloproteinase-2/9 expression and targeting homeobox D10 (28). Another study by Gong et al (29) revealed that miR-92b-3p inhibition prevented 
colorectal carcinoma cell proliferation, invasion, and migration by upregulating F-box with WD repeated domain-containing 7 (FBXW7). Notably, a previous study by Ma et al has reported miR-92b-3p was deregulated in PCa cells and this may be related with chemoresistance of tumor cells (17). Nevertheless, the expression of miR-92b-3p in PCa tissues and its clinical and functional role in PCa progression remain largely elusive. In the present study, miR-92b-3p was upregulated in PCa tissues and cell lines compared with normal tissues and normal cells, and the expression of miR-92b-3p was associated with PSA, bone metastasis, TNM stage and Gleason score of patients with $\mathrm{PCa}$, which suggested that miR-92b-3p might be involved in PCa development. In addition, the recorded 5-year follow-up survival information analyzed by Kaplan-Meier survival curves demonstrated that the patients with higher miR-92b-3p expression had shorter overall survival rates compared with those patients with lower miR-92b-3p levels. Besides, in patients with PCA who had positive bone metastasis, high levels of miR-92b-3p were also associated with shorter overall survival time when compared to patients with low levels of miR-92b-3p. Although the survival time in patients without bone metastasis was also shorter when they had high expression of miR-92b-3p, but the difference did not reach statistically significance, which may due to the limited sample size. In addition, Cox regression analysis further revealed that miR-92b-3p was an independent prognostic factor for $\mathrm{PCa}$. According to these findings of the present study, miR-92b-3p may serve as a biomarker for $\mathrm{PCa}$ prognosis.

A number of studies have provided evidence for the therapeutic potential of miRNAs in a wide variety of human cancers, including PCa $(30,31)$. The proposed functional miRNAs exert therapeutic potential by regulating tumor cell biological processes, such as cell proliferation, migration and invasion (32). Thus, cell experiments were conducted in the present study to investigate the functional role of miR-92b-3p in PCa progression. The expression of miR-92b-3p was regulated by miR-92b-3p mimic or inhibitor following transfection. The MTT assay findings of the present study revealed that the overexpression of miR-92b-3p promoted cell proliferation, migration and invasion, while the downregulation of miR-92b-3p led to opposite results, which suggested that miR-92b-3p may function as an oncogene in PCa progression. The oncogenic role of miR-92b-3p has also been demonstrated in other malignancies, such as colorectal carcinoma and gastric cancer $(28,29)$. FBXW7 has been identified as a tumor suppressor in the progression of non-small cell lung carcinoma (NSCLC), and was related with the chemoresistance of NSCLC $(33,34)$. Whether miR-92b-3p could regulate FBXW7 in NSCLC is unclear, and whether miR-92b-3p could be involved in the chemoresistance of NSCLC through targeting FBXW7 is also uncertain.

There were some limitations to the present study. First, the sample size was relatively small, which may limit the accuracy of analysis results, such as the Kaplan-Meier survival analysis for patients with $\mathrm{PCa}$ without bone metastasis. Second, although the potential target genes of miR-92b-3p were identified, the exact target of miR-92b-3p in PCa was not explored. Thus, the results and conclusion should be confirmed and improved by further studies with a larger study population and mechanism-related investigations.
In conclusion, the present study found that miR-92b-3p was upregulated in PCa tissues and cells compared with normal controls. The overexpression of miR-92b-3p predicted poor prognosis of patients with $\mathrm{PCa}$ and can be used as an independent prognostic biomarker. Downregulation of miR-92b-3p is able to suppress cell proliferation, migration and invasion of PCa cells. Based on these findings, miR-92b-3p may act as a potential therapeutic target for patients with PCa.

\section{Acknowledgements}

Not applicable.

\section{Funding}

No funding was received.

\section{Availability of data and material}

All data analyzed during this study are included in the published article.

\section{Authors' contributions}

GW, BC and WL conducted this study, analyzed the clinical data and wrote the manuscript. RJ and BT performed the cell experiments and analyzed the corresponding data. All authors have read and approved the final manuscript.

\section{Ethics approval and consent to participate}

All patients provided signed informed consent and the present study received approval from the Ethics Committee of Shengli Oilfield Central Hospital (Dongying, China; approval no. SLYTh100219).

\section{Patient consent for publication}

Consent for publication was obtained from the patients.

\section{Competing interests}

The authors declare that they have no competing interests.

\section{References}

1. Castillejos-Molina RA and Gabilondo-Navarro FB: Prostate cancer. Salud Publica Mex 58: 279-284, 2016.

2. Torre LA, Bray F, Siegel RL, Ferlay J, Lortet-Tieulent J and Jemal A: Global cancer statistics, 2012. CA Cancer J Clin 65: 87-108, 2015.

3. Pu YS, Chiang HS, Lin CC, Huang CY, Huang KH and Chen J: Changing trends of prostate cancer in Asia. Aging Male 7: 120-132, 2004.

4. Kimura T and Egawa S: Epidemiology of prostate cancer in Asian countries. Int J Urol 25: 524-531, 2018.

5. Ferlay J, Soerjomataram I, Dikshit R, Eser S, Mathers C, Rebelo M, Parkin DM, Forman D and Bray F: Cancer incidence and mortality worldwide: Sources, methods and major patterns in GLOBOCAN 2012. Int J Cancer 136: E359-E386, 2015.

6. Perdana NR, Mochtar CA, Umbas R and Hamid AR: The risk factors of prostate cancer and its prevention: A literature review. Acta Med Indones 48: 228-238, 2016. 
7. Redman JM, Gulley JL and Madan RA: Combining immunotherapies for the treatment of prostate cancer. Urol Oncol 35: 694-700, 2017.

8. Gasnier A and Parvizi N: Updates on the diagnosis and treatment of prostate cancer. Br J Radiol 90: 20170180, 2017.

9. Powell Gray B, Kelly L, Ahrens DP, Barry AP, Kratschmer C, Levy $M$ and Sullenger BA: Tunable cytotoxic aptamer-drug conjugates for the treatment of prostate cancer. Proc Natl Acad Sci USA 115: 4761-4766, 2018

10. Rupaimoole R, Calin GA, Lopez-Berestein G and Sood AK: miRNA deregulation in cancer cells and the tumor microenvironment. Cancer Discov 6: 235-246, 2016.

11. Esquela-Kerscher A and Slack FJ: Oncomirs-microRNAs with a role in cancer. Nat Rev Cancer 6: 259-269, 2006.

12. Markou A, Zavridou M and Lianidou ES: miRNA-21 as a novel therapeutic target in lung cancer. Lung Cancer (Auckl) 7: 19-27, 2016.

13. Wu HM and Kim SG: miRNA-324, a potential therapeutic target for paracetamol-induced liver injury. Stem Cell Investig 3: 67, 2016.

14. Chen JY, Xu LF, Hu HL, Wen YQ, Chen D and Liu WH: miRNA-215-5p alleviates the metastasis of prostate cancer by targeting PGK1. Eur Rev Med Pharmacol Sci 24: 639-646, 2020.

15. Zhang $X$ and $\mathrm{Wu}$ J: Prognostic role of microRNA-145 in prostate cancer: A systems review and meta-analysis. Prostate Int 3: 71-74, 2015.

16. Long M, Zhan M, Xu S, Yang R, Chen W, Zhang S, Shi Y, He Q, Mohan M, Liu Q and Wang J: miR-92b-3p acts as a tumor suppressor by targeting Gabra3 in pancreatic cancer. Mol Cancer 16: 167, 2017.

17. Ma H, Wang LY, Yang RH, Zhou Y, Zhou P and Kong L: Identification of reciprocal microRNA-mRNA pairs associated with metastatic potential disparities in human prostate cancer cells and signaling pathway analysis. J Cell Biochem 120: 17779-17790, 2019.

18. Epstein JI, Egevad L, Amin MB, Delahunt B, Srigley JR and Humphrey PA; Grading Committee: The 2014 international society of urological pathology (ISUP) consensus conference on gleason grading of prostatic carcinoma: Definition of grading patterns and proposal for a new grading system. Am J Surg Pathol 40: 244-252, 2016.

19. Singletary SE, Allred C, Ashley P, Bassett LW, Berry D, Bland KI, Borgen PI, Clark GM, Edge SB, Hayes DF, et al: Staging system for breast cancer: Revisions for the 6th edition of the AJCC cancer staging manual. Surg Clin North Am 83: 803-819, 2003.

20. Livak KJ and Schmittgen TD: Analysis of relative gene expression data using real-time quantitative PCR and the 2(-Delta Delta C(T)) method. Methods 25: 402-408, 2001.

21. Zhao Y, Song Y, Yao L, Song G and Teng C: Circulating microRNAs: Promising biomarkers involved in several cancers and other diseases. DNA Cell Biol 36: 77-94, 2017.

22. Steinberg BA and Fang JC: Long-term outcomes of acute heart failure: Where are we now? J Am Coll Cardiol 70: 2487-2489, 2017.

23. Zhao L, Yu H, Yi S, Peng X, Su P, Xiao Z, Liu R, Tang A, Li X, Liu F and Shen S: The tumor suppressor miR-138-5p targets PD-L1 in colorectal cancer. Oncotarget 7: 45370-45384, 2016.
24. Wang L, Li B, Zhang L, Li Q, He Z, Zhang X, Huang X, Xu Z, Xia Y, Zhang Q, et al: miR-664a-3p functions as an oncogene by targeting Hippo pathway in the development of gastric cancer. Cell Prolif 52: e12567, 2019.

25. Gong M, Chen C, Zhao H, Sun M and Song M: miR-506 suppresses cervical cancer cell proliferation both in vitro and in vivo. Neoplasma 65: 331-338, 2018.

26. Zhang Y, Zhang D, Lv J, Wang S and Zhang Q: miR-410-3p promotes prostate cancer progression via regulating PTEN/AKT/mTOR signaling pathway. Biochem Biophys Res Commun 503: 2459-2465, 2018.

27. Kachakova D, Mitkova A, Popov E, Popov I, Vlahova A, Dikov T, Christova S, Mitev V, Slavov C and Kaneva R: Combinations of serum prostate-specific antigen and plasma expression levels of let-7c, miR-30c, miR-141, and miR-375 as potential better diagnostic biomarkers for prostate cancer. DNA Cell Biol 34: 189-200, 2015.

28. Li C, Huo B, Wang Y and Cheng C: Downregulation of microRNA-92b-3p suppresses proliferation, migration, and invasion of gastric cancer SGC-7901 cells by targeting Homeobox D10. J Cell Biochem 120: 17405-17412, 2019.

29. Gong L, Ren M, Lv Z, Yang Y and Wang Z: miR-92b-3p promotes colorectal carcinoma cell proliferation, invasion, and migration by inhibiting FBXW7 in vitro and in vivo. DNA Cell Biol 37: 501-511, 2018.

30. Arisan ED, Rencuzogullari O, Freitas IL, Radzali S, Keskin B, Kothari A, Warford A and Uysal-Onganer P: Upregulated Wnt-11 and miR-21 expression trigger epithelial mesenchymal transition in aggressive prostate cancer cells. Biology (Basel) 9: 52, 2020.

31. Krebs M, Solimando AG, Kalogirou C, Marquardt A, Frank T, Sokolakis I, Hatzichristodoulou G, Kneitz S, Bargou R, Kübler H, et al: miR-221-3p regulates VEGFR2 expression in high-risk prostate cancer and represents an escape mechanism from sunitinib in vitro. J Clin Med 9: 670, 2020.

32. Zheng C, Guo K, Chen B, Wen Y and Xu Y: miR-214-5p inhibits human prostate cancer proliferation and migration through regulating CRMP5. Cancer Biomark 26: 193-202, 2019.

33. Hidayat M, Mitsuishi Y, Takahashi F, Tajima K, Yae T, Miyahara K, Hayakawa D, Winardi W, Ihara H, Koinuma Y, et al: Role of FBXW7 in the quiescence of gefitinib-resistant lung cancer stem cells in EGFR-mutant non-small cell lung cancer. Bosn J Basic Med Sci 19: 355-367, 2019.

34. Xiao G, Li Y, Wang M, Li X, Qin S, Sun X, Liang R, Zhang B, Du N, Xu C, et al: FBXW7 suppresses epithelial-mesenchymal transition and chemo-resistance of non-small-cell lung cancer cells by targeting snail for ubiquitin-dependent degradation. Cell Prolif 51: e12473, 2018.

This work is licensed under a Creative Commons Attribution-NonCommercial-NoDerivatives 4.0 International (CC BY-NC-ND 4.0) License. 